Research Article

\title{
Signature Verification Using Young's Lattice Grid Modeling
}

\author{
A. Hadjipanteli", E. N. Zois and A. Nassiopoulos. \\ Department of Electronic Engineering, Technological and Educational Institute of Athens \\ Egaleo, 12210 Greece.
}

Received 30 September 2015; Accepted 15 September 2016

\begin{abstract}
A promising off-line signature verification system based on grid based feature extraction is designed and evaluated. This work begins with a brief overview of the various stages which comprise a signature verification system. Next, the Young's lattice grid feature extraction concept is described in order to represent and model the primitive occurrences of handwritten signature. Two verification approaches have been applied based on the minimum distance classifier and the binary support vector machine. System evaluation was performed by using the MCYT-75 signature database. Experimental results based on the database are presented and the method seems promising; although further study is required.
\end{abstract}

Keywords:Signature Verification, Grid Features, Young's Lattice, Support Vector Machine.

\section{Introduction}

It has been accepted beyond any reasonable doubt that the handwritten signature remains one of the most common and acceptable ways for a person to declare his/hers identity. In order to facilitate this, signal/image processing and pattern recognition techniques are combined in order to provide an Automated Handwritten Signature Verification Systems (ASVS) in many application areas including civilian ones [1-4]. ASVS are broadly divided into two major categories based on the method that the signature is acquired. Both of these categories must manage the fact that the process of generating handwritten signatures, even when they are devised from a well-trained authentic writer, will convey natural discrepancies, defined as intra-writer variability [5]. A frequently used figure of merit which is enabled in order to describe the efficiency of ASVS is the equal error rate (EER) which is calculated from the ROC or DET plots of both types of error rates namely Type I error or false positive and Type II error or false negative.

The objective target of an offline ASVS is to transform the handwritten image into a mathematical measurable space where it will be characterized by its corresponding features [6]. Next, these are presented into classifiers which will decide, after appropriate training and testing procedures, if a signature under query belongs or not to the writer under claim [7-8]. According to the experimental protocol followed, there are two major approaches which have been applied to off-line ASVS namely writer dependent (WD) and writer-independent (WI). The WD approach uses an atomic classifier for each writer while the WI approach uses a classifier to match each input questioned signature to one or more reference signatures, and a single classifier is trained for all writers [9-10]. Feature extraction is considered by far the most puzzling task when ASVS are developed. A significant feature extraction viewpoint, exploit the signature

- E-mail address: argyrishadjipanteli@gmail.com

ISSN: $1791-2377$ @ 2016 Eastern Macedonia and Thrace Institute of Technology. All rights reserved. c features by using a coarse or fine detail grid imposed upon the image. Among others, examples of grid based feature extraction can be found in the work provided by references [10-19].

In this work another novel grid-based feature extraction method is presented and evaluated by using binary grid masks. These are simple binary formulations of the Young's Lattices and their corresponding absolute appearances along with first order appearances inside a predefined image provide the signature features. In the case study presented here a WD verification scheme is followed which is comprised of the training and testing phase. Verification results have been drawn with the use of a well-established database namely the MCYT-75 [20], [21] and the false acceptance (Type I), false rejection (Type II) and the equal error rate (EER) figures of merit.

The rest of this work is organized as follows: Section 2 provides the database details along with the preprocess procedure. Section 3 presents the feature extraction algorithm while section 4 deals with the applied experimental verification protocol. Section 5 presents the comparative evaluation results while section 6 draws the conclusions.

\section{Database - Preprocessing}

\subsection{Database}

The proposed feature extraction modeling has been studied with the use of the MCYT-75 database [20], [21]. It consists of 75 writers, were each of them is modelled by 15 genuine signatures and 15 simulated forgeries. Thus, the total number of signature is 2250. During the experimental process, two schemes of randomly selected training and testing samples were used for comparison with the outcomes of contemporary research in the field. In the first scheme, 5 genuine and 5 simulated-forgery reference samples per writer are used, while in the second scheme 10 genuine and 
10 simulated forgery reference samples are used. The remaining samples are used for testing.

\subsection{Preproccesing}

In order to produce the binary form of the acquired signatures the following preprocessing steps have been carried out: thresholding using the well known method of Otsu's [23], skeletonization [6], cropping and segmentation into various partitions in order to exploit fine and coarse level detail of the signature. This procedure is expected to reduce a number of side effects of the writing instruments variations like the distribution of the ink. The features are extracted either from the entire signature or from segments of signature's with the use of the equimass sampling grid method [14]. Equimass sampling grid segmentation provides strips of the signature with uniform size of signature pixels instead of the trivial distance grid segmentation which provides segments of equal area.

The segmentation procedure resulted into the following plans: a) the image was left as it is $(1 \times 1$ image $), b)$ the image was divided vertically $(1 \times 2$ image $), c)$ divided horizontally $(2 \times 1$ image $)$ and d) divided horizontally and vertically $(2 \times 2$ image). Proper attention was given in order for each part of the divided image to have an equal number of pixels.

\section{Feature Extraction}

\subsection{Young Lattices}

The feature extraction is based upon the existence of Young's lattices defined as a partially ordered set $\mathrm{Y}$ formed by all integer partitions ordered by inclusion of their Young diagrams [25]. This partially ordered set is formed by all integer partitions. It can be represented by a Hasse diagram where all elements within the same class are at the same level. Moreover the Young lattices are a graphical representation of numbers. The larger the number, the more representations can exist. Figure 1, presents the idea of Young's lattice for the integer partition of one up to eight.

\subsection{Feature Extraction}

Using the aforementioned Young's lattice modeling, two feature sets were produced namely the F1 and F2 set. In addition the F3 set arises from the union of the F1 and F2 sets. At this particular case, the Young's lattice which was enabled lie in the space of [1-8] as it is provided in Figure 1 also. This grid has 66 number representations and more than 200 links between them.

The feature extraction process utilizes the binary image provided from the preproccesing stage which is scanned vertically in order to identify signature pixels. Iteratively any of the Young's lattices elements along with its first order appearance is detected inside this $8 \times 8$ image window box. The search strategy begins from the higher order lattices and it is descending. As soon as it finds a match, it finds and all the appearances which are present either in an upward or downward manner. The procedure repeats until the entire signature image pixels are encoded.

\section{Verification}

In order to evaluate the performance of the proposed features, a comparative study is carried out by means of two well established classification schemes. The Minimum Distance Classifier (MDC) and the Support Vector Machine (SVM).

\subsection{Minimum Distance Classifier}

It can linearly classify data of two or more classes with relatively good accuracy and in a very short time [7]. For finding the distance uses either Euclidean distance or Mahalanobis distance. In this particular algorithm was used the second method. The Mahalanobis distance [22] is:

$$
D_{M}(x)=\frac{D_{M}(x)=\sqrt{(x-\mu)^{T} \Sigma^{-1}(x-\mu)}}{\sqrt{(x-\mu)^{T} \Sigma^{-1}(x-\mu)}}
$$

where the observation is a n-multidimensional feature vector $x=\left(x_{1}, x_{2}, \ldots, x_{n}\right)^{T} x=\left(x_{1}, x_{2} \cdots, x_{N}\right)^{T}$ derived from an observation group with parameters the associated mean values of each feature component $\mu=\left(\mu_{1}, \mu_{2}, \ldots \mu_{N}\right)^{T}$ along with its $\mu=\left(\mu_{1}, \mu_{2} \cdots, \mu_{N}\right)^{T}$ Scovariance matrix $\Sigma$ [26].

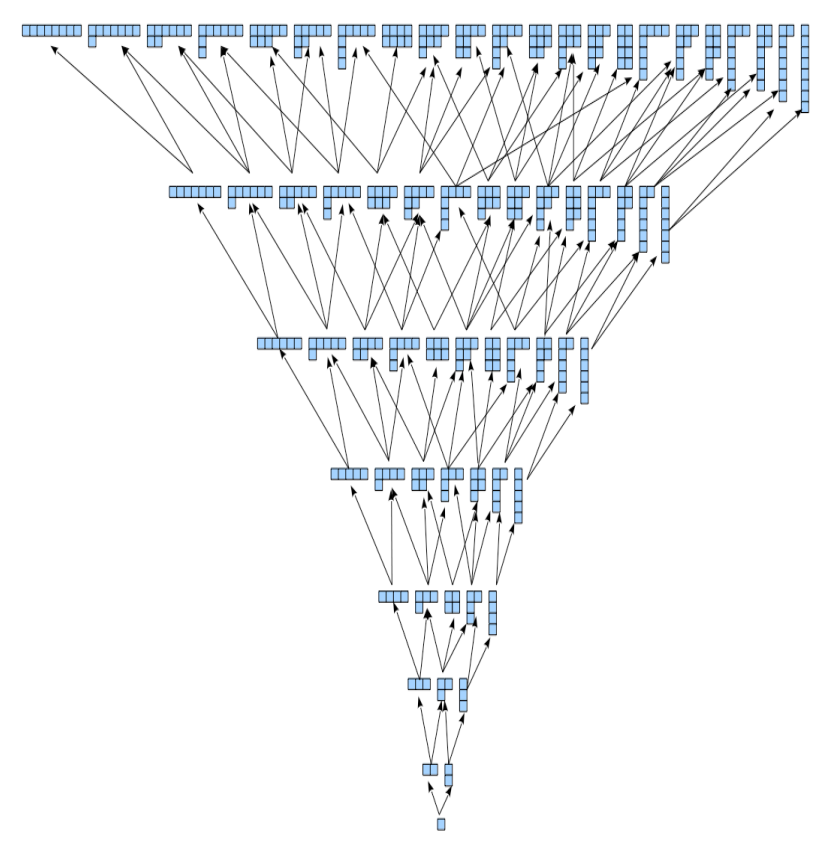

Fig. 1. Young lattice and corresponding grids.

\subsection{Support Vector Machine}

Support Vector Machines (SVM) [7] are another contemporary type of classifier with significant greater accuracy when it is compared to HMM's, and/or K-NNs. SVM's can be used either for separable or not classes.

\section{Results}

As it has been already mentioned, the feature extraction was based on Young lattices and for the verification the Minimum Distance Classifier and the SVM were used. Each algorithm was employed for all of the three feature sets. In addition, both of the verification algorithms were employed 5 times for each feature set and image division in order to establish reliable results.

From the extracted results the Type I error or false positive and Type II error or false negative were studied. Type I error presents the number of forgeries that were accepted as genuine and Type II error, presents the genuine samples that were rejected as forgeries. Finally the Average Error is calculated for each writer and consequently for the entire database. 


\subsection{Two Class Minimum Distance Classifier}

There were 5 random samples used for training which automatically created a testing set of 20 signatures (10 genuine, 10 forgeries). The results are presented at Table 1 . For each image segmentation the minimum, maximum and the mean value for every writer were taken and presented. After that the mean values for the whole database were extracted. It is worth mentioning that the best results are provided for the $1 \times 1$ image segmentation and for the $\mathrm{F} 1$ set.

\subsection{Single Class Minimum Distance Classifier}

According to the discussion previously exposed there were 5 runs conducted for each set and each image. In Table 2 the extracted results are presented. As in the two class Minimum Distance Classifier case we observe that the best results were derived for the $1 \times 1$ image segmentation scheme and for the F1 set.

\subsection{Two Class Support Vector Machine}

In this case the same procedure with the Two Class Minimum Distance Classifier was used. Thus, 10 samples (5 genuine, 5 forgeries) were used for training and 20 samples (10 genuine, 10 forgeries) for testing.

Table 1. Two Class Minimum Distance Classifier

\begin{tabular}{c|c|c|c|c}
\hline \multicolumn{5}{c}{$\begin{array}{c}\text { Average Error } \\
\text { (\%) }\end{array}$} \\
\hline $\begin{array}{c}\text { Image } \\
\text { Segmentation Level }\end{array}$ & Set & MIN & MEAN & MAX \\
\hline $1 \times 1$ & & 12.67 & 23.47 & 35.33 \\
$1 \times 1 \& 1 \times 2$ & & 12.93 & 24.93 & 38.07 \\
$1 \times 1 \& 2 \times 1$ & F1 & 14.20 & 26.09 & 36.93 \\
$1 \times 1 \& 2 \times 2$ & & 15.20 & 25.84 & 37.00 \\
\hline $1 \times 1$ & & 14.80 & 25.01 & 35.80 \\
$1 \times 1 \& 1 \times 2$ & & 14.73 & 26.43 & 38.00 \\
$1 \times 1 \& 2 \times 1$ & F2 & 17.40 & 28.79 & 39.60 \\
$1 \times 1 \& 2 \times 2$ & & 17.00 & 28.03 & 38.40 \\
\hline $1 \times 1$ & & 11.40 & 22.97 & 35.13 \\
$1 \times 1 \& 1 \times 2$ & & 13.33 & 24.95 & 36.47 \\
$1 \times 1 \& 2 \times 1$ & F3 & 15.87 & 26.31 & 37.27 \\
$1 \times 1 \& 2 \times 2$ & & 16.07 & 26.53 & 36.73 \\
\hline
\end{tabular}

Table 2. Single Class Minimum Distance Classifier

\begin{tabular}{c|c|c|c|c}
\hline \multicolumn{5}{|c}{$\begin{array}{c}\text { Average Error } \\
\text { (\%) }\end{array}$} \\
\hline $\begin{array}{c}\text { Image } \\
\text { Segmentation Level }\end{array}$ & Set & MIN & MEAN & MAX \\
\hline $1 \times 1$ & & 12.19 & 20.65 & 30.96 \\
$1 \times 1 \& 1 \times 2$ & & 11.83 & 22.44 & 33.08 \\
$1 \times 1 \& 2 \times 1$ & F1 & 12.68 & 22.08 & 31.95 \\
$1 \times 1 \& 2 \times 2$ & & 14.32 & 23.74 & 34.16 \\
\hline $1 \times 1$ & & 13,93 & 22,66 & 32,33 \\
$1 \times 1 \& 1 \times 2$ & & 14,43 & 23,26 & 32,57 \\
$1 \times 1 \& 2 \times 1$ & F2 & 15,80 & 24,16 & 33,75 \\
$1 \times 1 \& 2 \times 2$ & & 15,51 & 25,43 & 36,08 \\
\hline $1 \times 1$ & & 12,41 & 20,96 & 31,09 \\
$1 \times 1 \& 1 \times 2$ & F3 & 12,68 & 22,27 & 32,60
\end{tabular}

\begin{tabular}{c|c|c|c|c}
\hline \multicolumn{5}{c}{$\begin{array}{c}\text { Average Error } \\
(\%)\end{array}$} \\
\hline $\begin{array}{c}\text { Image } \\
\text { Segmentation Level }\end{array}$ & Set & MIN & MEAN & MAX \\
\hline $1 \times 1 \& 2 \times 1$ & & 12,84 & 22,52 & 32,15 \\
$1 \times 1 \& 2 \times 2$ & & 15,08 & 24,69 & 35,49 \\
\hline
\end{tabular}

From the study of Table 3 it is clear that the $1 \times 1$ image for the F1 set provides the best results. In addition the results are consistent when compared with the Two Class Minimum Distance Classifier.

\subsection{Single Class Support Vector Machine}

For the single class SVM case 10 runs were conducted for each image and writer. The procedure of the testing sample and the training sample is similar to the one class minimum distance classifier. Again it is observed that the $1 \times 1$ image segmentation along with the F1 set provide the best results. In addition, SVM classifier seems to provide the more efficient results from all of the classifiers mentioned beforehand.

Table 3. Two Class Support Vector Machine

\begin{tabular}{c|c|c|c|c}
\hline \multicolumn{5}{c}{$\begin{array}{c}\text { Average Error } \\
\text { (\%) }\end{array}$} \\
\hline $\begin{array}{c}\text { Image } \\
\text { Segmentation Level }\end{array}$ & Set & MIN & MEAN & MAX \\
\hline $1 \times 1$ & & 07.37 & 13.77 & 21.53 \\
$1 \times 1 \& 1 \times 2$ & & 07.26 & 13.24 & 22.04 \\
$1 \times 1 \& 2 \times 1$ & F1 & 07.15 & 12.99 & 20.21 \\
$1 \times 1 \& 2 \times 2$ & & 07.83 & 14.05 & 21.29 \\
\hline $1 \times 1$ & & 11.25 & 18.46 & 26.95 \\
$1 \times 1 \& 1 \times 2$ & & 12.26 & 19.31 & 26.60 \\
$1 \times 1 \& 2 \times 1$ & F2 & 12.62 & 19.23 & 26.66 \\
$1 \times 1 \& 2 \times 2$ & & 13.40 & 19.84 & 28.00 \\
\hline $1 \times 1$ & & 0959 & 16.03 & 24.47 \\
$1 \times 1 \& 1 \times 2$ & & 11.70 & 18.01 & 25.74 \\
$1 \times 1 \& 2 \times 1$ & F3 & 11.00 & 18.05 & 26.53 \\
$1 \times 1 \& 2 \times 2$ & & 12.90 & 19.28 & 26.02 \\
\hline
\end{tabular}

\section{Conclusions}

In order to provide a more accurate view on the results a comparison with the work provided in [9] is made. More specific the comparison is being made for the results of the skilled forgeries. The average error ranges from $11 \%-16 \%$ and the minimum error from the Tables $1-4$ range from $7 \%$ $15 \%$. The average error has a range $13 \%-29 \%$. Given the simplicity of the feature extraction method, the results can be considered more than satisfying. However it must be noted that proper cross-validation procedures must take place in order to properly sustain our results.

Table 4. Single Class Support Vector Machine

\begin{tabular}{c|c|c|c|c}
\hline \multicolumn{5}{c}{$\begin{array}{c}\text { Average Error } \\
\text { (\%) }\end{array}$} \\
\hline $\begin{array}{c}\text { Image Segmentation } \\
\text { Level }\end{array}$ & Set & MIN & MEAN & MAX \\
\hline $1 \times 1$ & \multirow{2}{*}{ F1 } & 09.00 & 18.00 & 28.00 \\
$1 \times 2$ & & 10.00 & 18.00 & 29.00
\end{tabular}




\begin{tabular}{c|c|c|c|c}
\hline \multicolumn{5}{c}{$\begin{array}{c}\text { Average Error } \\
\text { (\%) }\end{array}$} \\
\hline $\begin{array}{c}\text { Image Segmentation } \\
\text { Level }\end{array}$ & Set & MIN & MEAN & MAX \\
\hline $2 \times 1$ & & 10.00 & 18.00 & 29.00 \\
$2 \times 2$ & & 11.00 & 18.00 & 28.00 \\
\hline $1 \times 1$ & & 11.00 & 80.00 & 31.00 \\
$1 \times 2$ & & 11.00 & 19.00 & 29.00 \\
$2 \times 1$ & \multirow{2}{*}{ F2 } & 12.00 & 20.00 & 31.00 \\
\cline { 1 - 1 } $2 \times 2$ & & 12.00 & 20.00 & 29.00 \\
\hline
\end{tabular}

\begin{tabular}{c|c|c|c|c}
\hline \multicolumn{5}{c}{$\begin{array}{c}\text { Average Error } \\
\text { \%) }\end{array}$} \\
\hline $\begin{array}{c}\text { Image Segmentation } \\
\text { Level }\end{array}$ & Set & MIN & MEAN & MAX \\
\hline $1 \times 1$ & & 10.00 & 19.00 & 30.00 \\
$1 \times 2$ & & 11.00 & 18.00 & 29.00 \\
$2 \times 1$ & F3 & 11.00 & 19.00 & 30.00 \\
$2 \times 2$ & & 11.00 & 19.00 & 29.00 \\
\hline
\end{tabular}

\section{References}

1. R. Plamondon and S. N. Srihari, "On-line and off-line handwriting recognition: A comprehensive survey," IEEE Transactions on Pattern Analysis and Machine Intelligence, vol. 22, pp. 63-84, 2000.

2. F. Leclerc and R. Plamondon, "Automatic Signature verification: the state of the art-1989-1993", International Journal of Pattern Recognition and Artificial Intelligence, vol. 8, pp. 643-660, 1994.

3. D. Impedovo, and G. Pirlo, "Automatic signature verification: The state of the art, "IEEE Transactions on Systems Man and Cybernetics" vol. 38, pp. 609-635, September 2008.

4. L. Batista, D. Rivard, R. Sabourin, E. Granger, and P. Maupin, "State of the art in off-line signature verification". In Verma, B., Blumenstein, M. (eds.) Pattern Recognition Technologies and Applications: Recent Advances, pp. 39-62, 2008.

5. M. C. Fairhurst, "Signature verification revisited: promoting practical exploitation of biometric technology", Electron. Commun. Eng. J., vol. 9, pp. 273-280, 1997.

6. R. C. Gonzalez and R. E. Woods, "Digital Image processing", Addison Wesley, Reading, 1992.

7. S. Theodoridis and K. Koutroumbas, "Pattern Recognition", Academic Press, 2009.

8. R. O. Duda and P. E. Hart, Pattern classification. New York: John Wiley and Sons, 2001.

9. D. Bertolini, L. S. Oliveira, E. Justino, and R. Sabourin, "Reducing forgeries in writer-independent off-line signature verification through ensemble of classifiers". Pattern Recognition, vol. 43, pp. 387-396, 2010.

10. D. Rivard, E. Granger and R. Sabourin, "Multi feature extraction and selection in writer independent off-line signature verification", International Journal on Document Analysis and Recognition, vol. 16, pp. 83-103, 2013.

11. V. K. Madasu, and B. Lovell. "An Automatic Off-line Signature Verification and Forgery Detection System", in Verma, B., Blumenstein, M. (eds.) Pattern Recognition Technologies and Applications: Recent Advances, pp. 63-89, 2008.

12. J. F. Vargas, M. A. Ferrer, C. M. Travieso and J. B. Alonso, "Offline signature verification based on grey level information using texture features", Pattern Recognition, vol. 44, pp. 375-385, 2011.

13. R. Kumar, J. D. Sharma, and B. Chanda, "Writer independent offline signature verification using surroundedness feature", Pattern Recognition Letters, vol. 33, pp. 301-308, 2012.

14. D. Impedovo, G. Pirlo, L. Sarcinella, E. Stasolla, and C. A. Trullo, "Analysis of Stability in Static Signatures using Cosine Similarity", in: Proc of International Conference on Frontiers in Handwriting Recognition, pp. 231-235, 2012.

15. B. H. Shekar, and R. K. Bharathi, "LOG-Grid based off-line signature verification", in Fourth International Conference on signal and image processing, 2012. S. Mohan, S., Kumar, S.S. (eds), LNEE 222, pp. 321-330, 2013.

16. J. P. Swanepoel, and J. Coester. "Off-line signature verification using flexible grid features and classifiers fusion", in International Conference on Frontiers in Handwriting Recognition, pp. 297-302, 2012

17. M. K. Kalera, S. Shrihari and A. Xu, "Offine line signature verification using distance statistics", International Journal of Pattern Recognition and Artificial Intelligence, vol. 18, pp. 13391360, 2005.

18. A. Gilperez, F. Alonso-Fernandez, S. Pecharroman, J. FierrezAguilar, and J. Ortega-Garcia, "Off-line signature verification using contour features", in International Conference on Frontiers in Handwriting Recognition ICFHR 2008.

19. M. Parodi, J. C. Gomez, and A. Belaid, "A circular grid-based rotation invariant feature extraction approach for off-line signature verification", in 11th International Conference on Document Analysis and Recognition, pp. 1289-1293, 2011.

20. J. Fierrez-Aguilar, N. Alonso-Hermira, G. Moreno-Marquez and J. Ortega-Garcia, "An off-line signature verification system based on fusion of local and global information", Workshop on Biometric Authentication LNCS 3087, Springer, pp. 295-306, May 2004.

21. J. Ortega-Garcia, J. Fierrez-Aguilar, D. Simon, J. Gonzalez, M. Faunder-Zanuy, V. Espinosa, A. Statue, I. Hernaez, J.-J. Igarza, C. Vivaracho, D. Escudero and Q.-I. Moro, "MCYT baseline corpus: a biomodal biometric database", IEE Proc.-Vis. Image Signal Process, vol 150, December 2003.

22. P. C. Mahalanobis, "On The Generalized Distance In Statistics", Proceedings of the National Institute of Sciences of India, vol 2, pp. 49-55, 1936

23. N. Otsu, "A Threshold Selection Method From Gray-Level Histograms", IEEE Transactions on systems, man and cypernetics, SMC-9, pp. 62-66, January 1979.

24. S. Theodoridis and K. Koutroumbas, "An Introduction to Pattern Recognition: A MATLAB Approach", Elsevier, 2010.

25. R. Suter, "Young's Lattice and Dihedral Symmetries", European Journal of Combinatronics, vol. 23, pp. 233-238, 2002

26. W. Feller, "An introduction to probability theory and its applications", Wiley, 1971. 